\title{
Dual antiplatelet therapy with aspirin and clopidogrel for acute high risk transient ischaemic attack and minor ischaemic stroke: a clinical practice guideline
}

In this article by Kameshwar Prasad and colleagues (BMJ 2018;363:k5130, doi:10.1136/bmj.k5130), the authors had initially suggested using aspirin as the preferred single antiplatelet agent after dual antiplatelet therapy. In a rapid response, Dr Akif Gani pointed out that other antiplatelet agents might be equally reasonable (www.bmj.com/content/363/bmj. $\mathrm{k} 5130 / \mathrm{rr}$ ). We have changed the wording in the "What you need to know" box and infographic to suggest that, after 10-21 days of dual antiplatelet therapy, patients should continue on single antiplatelet therapy alone.

We have also changed the wording of the bleeding outcomes in the infographic to be more specific about severity of bleeding. "Extracranial haemorrhage" has been changed to "Moderate or major bleeding," and "Extracranial bleeding" has been changed to "Minor bleeding."

Both print and online versions have been amended to reflect these changes. 